Published in final edited form as:

Neurobiol Dis. 2015 September ; 81: 1-3. doi:10.1016/j.nbd.2015.08.012.

\title{
Metals and neurodegeneration
}

\author{
Elias Aizenman and \\ Department of Neurobiology and Pittsburgh Institute for Neurodegenerative Diseases, University \\ of Pittsburgh School of Medicine, Pittsburgh, PA, USA
}

Pier G. Mastroberardino

Department of Genetics, Erasmus University Medical Center, Rotterdam, The Netherlands

\begin{abstract}
Metal biology is receiving an unprecedented level of attention from the field of neuroscience. As accumulating evidence continues to substantiate the essential function of metals in the healthy brain, studies exploring the causative role of metals in the molecular pathogenesis of neurological disorders are rapidly expanding. Further excitement stems from the positive outcome of very recent preventive trials to restore metal homeostasis in neurodegenerative diseases (Devos et al., 2014). Metals biology is therefore no longer relegated to a niche of specialist researchers and is instead arousing the interest of a broad audience.
\end{abstract}

This field, however, deserves and demands even more consideration and research attention. Our understanding of the precise mechanisms governing metals homeostasis in the brain is very rudimentary, and evidence is even weaker when the extraordinary complexity of this organ system is taken into account and the problem is approached at the level of different functional and anatomical domains. Consequently, the nexus between derangement of metals homeostasis and neuropathology is poorly understood and the pathobiology of certain rare diseases featuring pronounced alterations in metals homeostasis - for instance Neurodegeneration with Iron Accumulation in the Brain - remains largely uncharacterized. It is with these concepts in mind that we assembled this collection of reviews and original research articles, with the ultimate goal of giving further resonance to metals biology in the healthy and diseased brain. We believe that this special issue provides a timely and effective contribution, in which comprehensive reviews and exciting and provocative research articles cover crucial open questions in metal neurobiology.

The issue features studies providing novel functional and mechanistic insights on metals in neurodegeneration, also substantiating the concept that unbalance in different pools - e.g. extracellular versus intracellular - might result in opposite consequences. Gilad et al. (2015) investigate the role of zinc $\left(\mathrm{Zn}^{2+}\right)$ signaling in epileptic seizures induced by the excitotoxin kainic acid and unravel an adaptive mechanism enhancing the crucial inhibitory $\mathrm{K}^{+} / \mathrm{Cl}^{-}$ cotransporter 2 (KCC2) through the synaptic metabotropic $\mathrm{Zn}^{2+}$ receptor mZnR/GPR39. The authors propose that synaptic $\mathrm{Zn}^{2+}$ released from glutamatergic terminals triggers the adaptive mechanism and might therefore exert a crucial neuroprotective function to restore 
inhibitory drive, preventing excitotoxicity during sustained seizures. Further intriguing evidence on the role of extracellular $\mathrm{Zn}^{2+}$ signaling comes from the study from PerezRosello et al. (2015). Here, the authors focused on the dorsal cochlear nucleus (DCN), which presents high levels of this metal, to gather insights on the poorly understood function of tonic $\mathrm{Zn}^{2+}$. Experimentally, the study takes advantage of a fast and high affinity $\mathrm{Zn}^{2+}$ chelator in a mutant mouse strain lacking the zinc transporter 3 (ZnT3) to demonstrate that depletion of bioavailable extracellular non-synaptic $\mathrm{Zn}^{2+}$ enhances spontaneous firing. This effect is blocked by application of a glycine receptor antagonist and therefore depends upon modulation of tonic glycinergic inhibition. Overall, the authors suggest that tonic zinc constitutes a powerful break of spontaneous firing of DCN and may protect against a pathological escalation of spontaneous activity, as for instance observed in tinnitus. A neuroprotective role for $\mathrm{Zn}^{2+}$ also emerges from the study of McAllum et al. (2015) exploring the effects of administration of complexed $\mathrm{Zn}^{2+}$ in mice overexpressing mutant $\mathrm{Cu}, \mathrm{Zn}$ superoxide dismutase (SOD1), modeling amyotrophic lateral sclerosis (ALS). In this experimental paradigm, $\mathrm{Zn}^{2+}$ improves metallation of mutant SOD1 - which is otherwise compromised in this pathogenic variant - ameliorating locomotor symptoms, and extending life span via a mechanism that progresses through improved copper delivery. The work of Granzotto and Sensi (2015) focuses instead on intra-cellular $\mathrm{Zn}^{2+}$ and substantiates its central role in excitotoxicity. The authors analyzed the metal influence on the dynamics of intracellular excitotoxic alterations of the metal and sought specific evidence by comparing NMDA effects in two striatal subpopulations: NMDA-insensitive nNOS-positive neurons and vulnerable nNOS-negative ones. The authors demonstrate that ROS-dependent release of intracellular $\mathrm{Zn}^{2+}$ acts synergistically with $\mathrm{Ca}^{2+}$ and is essential to induce irreversible mitochondrial damage and neuronal death. Finally, the work from Xu et al. (2015) investigates the potential pathogenic role of $\mathrm{Zn}^{2+}$ in Alzheimer's disease (AD) by exploring the effects of this metal on the stability of apolipoprotein E (ApoE), whose gene features allelic variants resulting in isoforms that influence the onset and progression of the disease. The authors show that, in the presence of exogenous proteases, $\mathrm{Zn}^{2+}$ promotes proteolysis of synthetic ApoE in an isoform-specific manner and consequently propose that the alterations in zinc homeostasis observed in $\mathrm{AD}$ might favor the formation of ApoE products involved in neurodegeneration.

$\mathrm{AD}$ is also the subject of a detailed review from Meadowcroft et al. (2015) summarizing our current understanding of the nexus between perturbations in iron homeostasis and two major pathogenic events associated with this devastating disease: formation of beta-amyloid plaques and hyperphosphorylated tau tangles. The authors discuss these elements in the context of the principal contributing mechanisms in $\mathrm{AD}$, seeking a unifying perspective that might integrate the roles of amyloid pathology and iron dysregulation. The article from Ghosh et al. (2015) provides a broader and more general discussion on the neurological consequences caused by iron balance by reviewing studies involving mouse models mutant for Iron regulatory proteins 1 and 2 (Irp1 and Irp2). These are cytosolic proteins crucial to regulate the expression of iron metabolism genes at the translational level and mice lacking Irp1 and/or Irp 2 fail to fine-tune their transcriptome to optimally preserve iron homeostasis. The review provides an excellent overview of iron metabolism, describes the different Irp strains generated thus far, elaborates on the progressive neurological defects observed in 
Irp2 mutants, and highlights how general defects in the iron regulating molecular machinery may impact the nervous system.

Two research articles are focused on Parkinson disease (PD). PD was among the first forms of chronic neurodegeneration found to be associated with perturbation of metal homeostasis, as reported in the seminal study of Hallgren and Sourander (1958) describing increased iron levels in the substantia nigra (SN) of PD patients. In PD, however, anomalies in metal homeostasis are not confined to iron and involve other elements that received only marginal attention, and whose contribution is far less understood. The articles in this issue approach the problem from the perspective of gene-environment interaction in PD etiopathology and investigate anomalies induced by copper. The work by Anandhan et al. (2015) sheds light on the mechanisms underlying copper toxicity in dopaminergic neurons and on the synergistic and detrimental interactions between this metal and alpha-synuclein. The study concludes that this synergism induces cell death via the perturbation of the ubiquitin-proteasome and autophagic systems, albeit independently from protein aggregation. The study from Aboud et al. (2014) explores the synergism between complete loss-of-function of parkin caused by homozygous mutations in the PARK2 gene and exposure to metals. The rationale of the approach stems from the incomplete penetrance of PARK2 loss-of-function mutations, which causes high intra-familial and inter-familial variability in ages of onset, and therefore reinforces the role of environmental exposure in eliciting pathogenesis. The authors show that inducible pluripotent stem cells (iPSC) obtained from two siblings with homozygous PARK2 loss-of-function and reprogrammed into neuroprogenitor cells exhibit increased susceptibility to copper and cadmium, therefore substantiating the concept that exposure to these metals might result in increased risk for PD.

How the different cellular populations constituting the nervous system handle iron in neurological diseases is still dimly understood, and this series features two articles investigating this important topic. Zarruk et al. (2015) studied the expression and the cellular localization of proteins involved in cellular iron influx, storage, and efflux in two different mouse models of experimental autoimmune encephalomyelitis (EAE). The study demonstrates that during EAE progression iron accumulates in the spinal cord and that accumulation is localized in macrophages and microglia, but not in the astrocytes that can recycle iron efficiently from sites of EAE lesions. Thomsen et al. (2015) also demonstrate iron accumulation in macrophages and microglia in a model of excitotoxicity based on the administration of the glutamate agonist ibotenic acid, which also triggers neuroinflammation. Interestingly, the authors also describe an increase of the master iron regulating hormone hepcidin following excitotoxic lesion.

The collection also gives resonance to orphan diseases and features manuscripts investigating or discussing to the role of metals in their pathogenesis. Maccarinelli et al. (2015) present a novel mouse model of neuroferrinopathy, a rare condition that belongs to the group of Neurodegeneration with Brain Iron Accumulation (NBIA) disorders, which is comprehensively reviewed in another article by Levi and Rovid in this series Levi and Rovida (2015). Maccarinelli, Pagani, and coworkers generated a mutant mouse that carries a pathogenic nucleotide insertion in the ferritin light chain (FTL) gene and that recapitulates essential features of the human disease. In these animals, the authors describe signs of 
oxidative stress in the brain and increased sensitivity of cultured primary neurons to iron overload and pro-oxidants. Additionally, the study reports the association of iron deposits with lipofuscin granules, particularly in the cerebellum and striatum; this observation, which also suggests some degree of anatomical specificity in the defects elicited by FTL mutations, prompted the authors to speculate a central role for lipofuscin in etiopathogenesis of human neuroferritinopathy. While further studies will be necessary to fully address this hypothesis, this article provides a new and very much needed tool to understand the mechanisms underlying neuroferrinopathy. Further observations on NBIA come from the study Santambrogio et al. (2015), which takes advantage of primary skin fibroblasts and neurons reprogramed from fibroblasts of patients harboring mutations in the PANK2 gene, and describes anomalies in redox balance, in iron homeostasis, and in bioenergetics. Zlatic et al. (2015) reviewed the molecular basis of neurodevelopmental and neurodegenerative defects in Menkes disease, a rare condition caused by mutations in the trans-Golgi coppertransporter P-ATPase, ATP7A. This article not only summarizes state-of-the-art knowledge about this disorder, but also challenges the predominant mechanistic hypothesis of pathogenesis - the oligoenzymatic hypothesis - providing a revised and more comprehensive version. The traditional theory claims that neurological manifestations typical of Menkes disease are attributable to malfunctioning of five copper-requiring enzymes in the brain: mitochondrial cytochrome oxidase $\mathrm{C}$, and four enzymes that acquire copper in the Golgi apparatus. The authors now suggest a broader interpretation according which the causes of neurological defects should be sought not only in the canonical five genes, but in all the genes belonging to the same ontological category: copper ion binding. The piece therefore provides a novel perspective of Menkes pathology and lays foundation for future mechanistic studies to test this extended version of the oligoenzymatic hypothesis. Squadrone et al. (2015) studied metals in another rare disease, ataxia-telengiectasia (A-T), a pediatric form of autosomal recessive cerebellar ataxia caused by mutations in the DNA repair gene ATM, and found alterations in circulating copper and zinc in patients. Although the question whether such changes reflect a pathogenic function of metal anomalies in this disease remains open, the study paves the road for further investigations to better understand the define the role of metals in A-T pathobiology and also establish an intriguing nexus between alterations in metal homeostasis and DNA repair. Further studies are warranted to elucidate mechanistic aspects of this exciting connection.

Finally, a set of articles focuses on prospective therapies targeting metals. The study from Lei et al. (2015) shows that clioquinol - a copper and zinc ionophore and a moderate iron chelator - selectively reverses iron accumulation in the brain of aged tau knockout mouse and ameliorates both parkinsonism and cognitive defects. Johanssen et al. (2015) describe how administration of the metal chaperone PBT2 preconditions neurons by inducing increase in cytosolic $\mathrm{Zn}^{2+}$, which in turn causes a sub-threshold elevation in $\mathrm{Ca}^{2+}$ levels protecting neurons from a subsequent a more severe NMDA toxic stimulus. A similar mechanism based on $\mathrm{Zn}^{2+}$-mediated preconditioning also underlies the neuroprotective effect of the anti-inflammatory drug indomethacin, a non-selective COX inhibitor that Lee and colleagues found to ameliorate ischemic injury in the brain (Lee et al., 2015).

In conclusion, increasing evidence substantiate a central role of metals in the etiopathogenesis of neurodegenerative diseases. Consequently, metal biology is receiving 
commensurate and escalating interest from the neuroscience community, as also highlighted by dedicated forum issues recently published by other Journals (Adlard and Chung, 2015; White et al., 2015). We believe that the collection we assembled encompasses topics of primary relevance in metal biology and provides examples of how metals can participate to a broad spectrum of neuro-degenerative disorders. We certainly hope that this series will serve well to both experienced metal investigators, as well as outsider scientists interested in approaching the field.

\section{References}

Aboud AA, et al. PARK2 patient neuroprogenitors show increased mitochondrial sensitivity to copper. Neurobiol Dis. 2014; 73C:204-212. [PubMed: 25315681]

Adlard PA, Chung RS. Editorial: the molecular pathology of cognitive decline: focus on metals. Front Aging Neurosci. 2015; 7:116. [PubMed: 26136683]

Anandhan A, et al. Overexpression of alpha-synuclein at non-toxic levels increases dopaminergic cell death induced by copper exposure via modulation of protein degradation pathways. Neurobiol Dis. 2015; 81:76-92. [PubMed: 25497688]

Devos D, et al. Targeting chelatable iron as a therapeutic modality in Parkinson's disease. Antioxid Redox Signal. 2014

Ghosh MC, et al. Iron misregulation and neurodegenerative disease in mouse models that lack iron regulatory proteins. Neurobiol Dis. 2015; 81:66-75. [PubMed: 25771171]

Gilad D, et al. Homeostatic regulation of KCC2 activity by the zinc receptor mZnR/GPR39 during seizures. Neurobiol Dis. 2015; 81:4-13. [PubMed: 25562657]

Granzotto A, Sensi SL. Intracellular zinc is a critical intermediate in the excitotoxic cascade. Neurobiol Dis. 2015; 81:25-37. [PubMed: 25940914]

Hallgren B, Sourander P. The effect of age on the non-haemin iron in the human brain. J Neurochem. 1958; 3:41-51. [PubMed: 13611557]

Johanssen T, et al. PBT2 inhibits glutamate-induced excitotoxicity in neurons through metal-mediated preconditioning. Neurobiol Dis. 2015; 81:176-185. [PubMed: 25697105]

Lee JY, et al. Indomethacin preconditioning induces ischemic tolerance by modifying zinc availability in the brain. Neurobiol Dis. 2015; 81:186-195. [PubMed: 25562658]

Lei $\mathrm{P}$, et al. Clioquinol rescues Parkinsonism and dementia phenotypes of the tau knockout mouse. Neurobiol Dis. 2015; 81:168-175. [PubMed: 25796563]

Levi S, Rovida E. Neuroferritinopathy: from ferritin structure modification to pathogenetic mechanism. Neurobiol Dis. 2015; 81:134-143. [PubMed: 25772441]

Maccarinelli F, et al. A novel neuroferritinopathy mouse model (FTL 498InsTC) shows progressive brain iron dysregulation, morphological signs of early neurodegeneration and motor coordination deficits. Neurobiol Dis. 2015; 81:119-133. [PubMed: 25447222]

McAllum EJ, et al. $\mathrm{Zn}^{\mathrm{II}}(\mathrm{atsm})$ is protective in amyotrophic lateral sclerosis model mice via a copper delivery mechanism. Neurobiol Dis. 2015; 81:20-24. [PubMed: 25766674]

Meadowcroft MD, et al. The relationship between iron dyshomeostasis and amyloidogenesis in Alzheimer's disease: two sides of the same coin. Neurobiol Dis. 2015; 81:49-65. [PubMed: 26303889]

Perez-Rosello T, et al. Tonic zinc inhibits spontaneous firing in dorsal cochlear nucleus principal neurons by enhancing glycinergic neurotransmission. Neurobiol Dis. 2015; 81:14-19. [PubMed: 25796568]

Santambrogio P, et al. Mitochondrial iron and energetic dysfunction distinguish fibroblasts and induced neurons from pantothenate kinase-associated neurodegeneration patients. Neurobiol Dis. 2015; 81:144-153. [PubMed: 25836419]

Squadrone $\mathrm{S}$, et al. Blood metal levels and related antioxidant enzyme activities in patients with ataxia telangiectasia. Neurobiol Dis. 2015; 81:162-167. [PubMed: 25882094] 
Thomsen MS, et al. Neurodegeneration with inflammation is accompanied by accumulation of iron and ferritin in microglia and neurons. Neurobiol Dis. 2015; 81:108-118. [PubMed: 25801802]

White AR, et al. Editorial: metals and neurodegeneration: restoring the balance. Front Aging Neurosci. 2015; 7:127. [PubMed: 26191002]

$\mathrm{Xu} \mathrm{H}$, et al. Zinc affects the proteolytic stability of Apolipoprotein E in an isoform-dependent way. Neurobiol Dis. 2015; 81:38-48. [PubMed: 26117305]

Zarruk JG, et al. Expression of iron homeostasis proteins in the spinal cord in experimental autoimmune encephalomyelitis and their implications for iron accumulation. Neurobiol Dis. 2015; 81:93-107. [PubMed: 25724358]

Zlatic S, et al. Molecular basis of neurodegeneration and neurodevelopmental defects in Menkes disease. Neurobiol Dis. 2015; 81:154-161. [PubMed: 25583185] 